\title{
Description of a Digital Health Platform for Emotional and Self-Management Support of Caregivers of Children Receiving Growth Hormone Treatment
}

\author{
Francisco José NÚÑEZ-BENJUMEA ${ }^{\mathrm{a}}$, Antonio De Arriba MUÑOZ ${ }^{\mathrm{b}}$, Marta Vara \\ CALLAU $^{\mathrm{b}}$ and Luis FERNANDEZ-LUQUE ${ }^{\mathrm{a}, 1}$ \\ a Adhera Health, Inc, Palo Alto, CA, USA \\ ${ }^{\mathrm{b}}$ Pediatric Endocrinology unit, Miguel Servet Children's University Hospital, \\ Zaragoza, Spain
}

\begin{abstract}
In this demo, we provide an overview of the digital platform ADHERA CARING which has been used for an intervention designed for emotional and selfmanagement support of caregivers of children receiving growth hormone treatment $(\mathrm{GHt})$. ADHERA CARING provides tailored emotional and self-management support to caregivers of children undergoing GHt to improve adherence to treatment through positive education, personalized motivational messages, and emotional support. This digital intervention has already been piloted in a clinical setting as part of an ongoing feasibility clinical study (NCT04812665).
\end{abstract}

Keywords. Digital Health, Growth Hormone, Behavioral Intervention, Caregiver

\section{Introduction}

Adherence to growth hormone treatment (GHt) among children is variable and remains a problem [1]. Prior research has been looking into digital health tools to support GHt treatment [2], that includes mobile applications [3], and more advanced digital health ecosystems that integrate mobile solutions with connected injection devices [4]. One of the key stakeholders to be supported are the caregivers, normally parents, of children that require long term pharmacological treatment since research shows the negative impact of stress on adherence and overall quality of life [5]. In this paper, we present a study exploring the feasibility of a mobile digital intervention for emotional and selfmanagement support of caregivers of children receiving GHt. The intervention relies on a digital health platform powered by a Health Recommender System [6].

\footnotetext{
${ }^{1}$ Corresponding Author, Luis Fernandez-Luque Adhera Health, Inc, Palo Alto, CA, USA; E-mail: luis.tromso@gmail.com.
} 


\section{2. mHealth-based digital intervention}

ADHERA CARING provides tailored emotional and self-management support to caregivers of children undergoing GHt to improve adherence to treatment through positive education, personalized motivational messages, and emotional support. Users follow an empowerment program with easy-to-digest educational, personalized, and actionable content included for them, and also perform mental well-being activities. Whenever a unit is tagged as read, its icon turns green to reflect the progress (Figure 1A). Within each category, the last educational unit is a quiz (Figure 1-B).

Motivational messages are delivered into an inbox in the app (Figure 1-C). Every time the app receives a new message, a push notification is triggered so that the user can access the message directly. Messages can be rated by users with a score from 1 to 5 stars. Messages are delivered by a health recommender system [6]. This algorithm works in two steps. The first step is a knowledge-based algorithm to filter incompatible messages with the user profile. The remaining messages are then passed to a collaborative filtering algorithm, that uses the demographic profile and the user ratings to compute a similarity measurement between the user profile and each message.

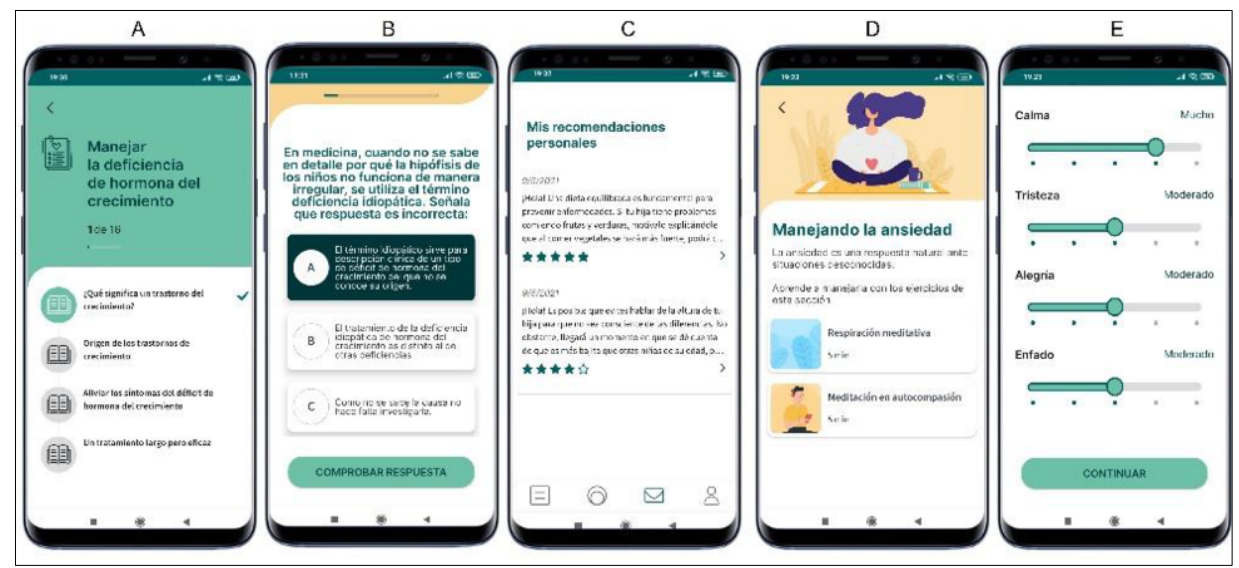

Figure 1. Screenshots of ADHERA CARING Platform

The mental well-being module (Figure 1-D) includes two exercises: 'Mindful breathing' and 'Self-compassion meditation'. These are audio-guided sessions that users can complete at their convenience. Before and after each session, the user is asked to complete a well-being assessment (Figure 1-E).

\section{Acknowledgements}

We would like to thank the rest of the team who contributed to this project and the caregivers for their participation.

Disclaimer: This investigator initiated study was financially supported by Merck Healthcare KGaA, Darmstadt Germany (CrossRef Funder ID: 10.13039/100009945). Merck reviewed the manuscript for medical accuracy only before submission. The authors are fully responsible for the content of this manuscript, and the views and opinions described in the publication reflect solely those of the authors. 


\section{References}

[1] Haverkamp F, Gasteyger C. A review of biopsychosocial strategies to prevent and overcome earlyrecognized poor adherence in growth hormone therapy of children. J Med Econ. 2011;14(4):448-57.

[2] Dimitri P, Fernandez-Luque L, Banerjee I, Bergadá I, Calliari LE, Dahlgren J, de Arriba A, Lapatto R, Reinehr T, Senniappan S, Thomas-Teinturier C. An eHealth Framework for Managing Pediatric Growth Disorders and Growth Hormone Therapy. Journal of Medical Internet Research. 2021 May 20;23(5):e27446.

[3] Fernandez-Luque L, Labarta JI, Palmer E, Koledova E. Content Analysis of Apps for Growth Monitoring and Growth Hormone Treatment: Systematic Search in the Android App Store. JMIR Mhealth Uhealth. 2020 Feb 18;8(2):e16208.

[4] Koledova E, Le Masne Q, Spataru A, Bagha M, Dixon D. Digital Health in the Management of Pediatric Growth Hormone Therapy - 10 Years of Developments. Stud Health Technol Inform. 2021 May 27;281:926-30.

[5] Armstrong ML, Duncan CL, Stokes JO, Pereira D. Association of caregiver health beliefs and parenting stress with medication adherence in preschoolers with asthma. J Asthma. 2014 May;51(4):366-72.

[6] Hors-Fraile S, Malwade S, Luna-Perejon F, Amaya C, Civit A, Schneider F, Bamidis P, Syed-Abdul S, Li YC, De Vries H. Opening the black box: Explaining the process of basing a health recommender system on the i-change behavioral change model. IEEE Access. 2019 Dec 4;7:176525-40. 